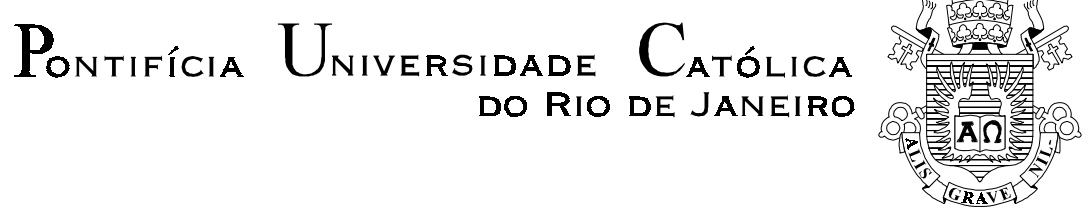

Roque André Ciufo Poeys

Efeitos de multipercursos atmosféricos em enlaces de microondas em visibilidade

Dissertação apresentada como requisito parcial para obtenção do título de Mestre pelo Programa de Pós-Graduação em Engenharia Elétrica da PUC-Rio.

Orientador: Luis Alencar Reis da Silva Mello

Rio de Janeiro 


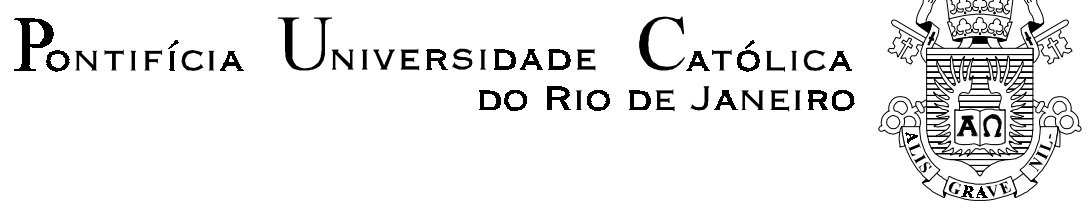

Roque André Ciufo Poeys

\title{
Efeitos de multipercursos atmosféricos em enlaces de microondas em visibilidade
}

Dissertação apresentada como requisito parcial para obtenção do título de Mestre pelo Programa de PósGraduação em Engenharia Elétrica da PUC-Rio. Aprovada pela Comissão Examinadora abaixo assinada.

\author{
Luiz Alencar Reis da Silva Mello \\ PUC-RIO \\ Rodolfo Sabóia Lima de Souza \\ PUC-RIO \\ Marta Pudwell Chaves de Almeida \\ Wings Telecom \\ Paulo Pereira Loureiro
Embratel
}

Erasmus Couto Brazil de Miranda

UCP

José Eugenio Leal

Coordenador Setorial do Centro Técnico Científico - PUC-Rio

Rio de Janeiro, 3 de setembro de 2004 
Todos os direitos reservados. É proibida a reprodução total ou parcial do trabalho sem autorização da universidade, do autor e do orientador.

\section{Roque André Ciufo Poeys}

Graduou-se em Engenharia Elétrica, ênfase em Sistemas Eletrônicos, em julho de 1989, na Universidade do Estado do Rio de Janeiro. Trabalha como Analista de Redes e Serviços de Telecomunicações na Embratel desde abril de 1999. É Professor de Telecomunicações da Escola Técnica Estadual Ferreira Viana desde março de 1998. Em fevereiro de 2002, iniciou no Centro de Estudos em Telecomunicações da Pontifícia Universidade Católica do Rio de Janeiro seu mestrado na área de eletromagnetismo aplicado.

Ficha Catalográfica

Poeys, Roque André Ciufo
Efeitos de multipercursos atmosféricos em
enlaces de microondas em visibilidade / Roque André
Ciufo Poeys ; orientador: Luis Alencar Reis da Silva Mello.
- Rio de Janeiro : PUC-Rio, Departamento de Engenharia
Elétrica, 2004.

94 f. ; $30 \mathrm{~cm}$

Dissertação (mestrado) - Pontifícia Universidade Católica do Rio de Janeiro, Departamento de Engenharia Elétrica.

Inclui referências bibliográficas

1. Engenharia elétrica - Teses. 2. Propagação multipercurso. 3. Desvanecimento seletivo. 4. Sistema rádio digital. 5. Predição de falha. I. Mello, Luiz Alencar Reis da Silva. II. Pontifícia Universidade Católica do Rio de Janeiro. Departamento de Engenharia Elétrica. III. Título. 


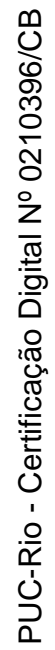

À minha esposa Rita, pela força do seu amor, pelo apoio constante e intenso e por sua dedicação e confiança durante todo o período de estudos e a nossa filha Lívia, o maior e o melhor presente de nossas vidas. 


\section{Agradecimentos}

Ao meu orientador Professor Luiz Alencar Reis da Silva Mello pelo apoio, estímulo e dedicação durante todas as etapas deste trabalho.

Ao engenheiro Sergio Pinho da Embratel pela ajuda, incentivo e dedicação sincera durante as etapas de pesquisa.

Aos engenheiros Lauro Fernando Santos Maia e Jorge de Souza Santos da Embratel pelo apoio e colaboração, imprescindíveis para a realização deste trabalho.

Ao professor Ney R. Dhein, por ter me dado a oportunidade de iniciar este mestrado.

Aos meus amigos e colegas da Embratel e do Cetuc, por todo apoio, colaboração e palavras de incentivo.

A todos os amigos e familiares que de uma forma ou de outra me estimularam ou me ajudaram.

A FAETEC e a PUC-Rio, pelos auxílios concedidos, sem os quais este trabalho dificilmente poderia ter sido realizado. 


\section{Resumo}

Poeys Ciufo, Roque André . Efeitos de multipercursos atmosféricos em enlaces de microondas em visibilidade. Rio de Janeiro, 2004. 94p. Dissertação de Mestrado - Departamento de Engenharia Elétrica, Pontifícia Universidade Católica do Rio de Janeiro.

As variações que ocorrem na estrutura da troposfera ao longo do tempo em relação à sua condição mediana provocam diversos fenômenos que fazem variar aleatoriamente o nível de sinal recebido num enlace rádio. Estas variações aleatórias são denominadas desvanecimentos. Os desvanecimentos são normalmente classificados em rápidos e lentos. Os desvanecimentos rápidos estão geralmente associados ao efeito de multipercurso atmosférico que é fortemente dependente da freqüência, sendo por isto denominados desvanecimentos seletivos, e são a principal causa de degradação do desempenho de enlaces rádio digitais de alta capacidade. Os modelos existentes para a caracterização estatística do desvanecimento por multipercurso são semi-empíricos e baseados em dados experimentais obtidos em regiões de clima temperado, acarretando uma má estimativa quando aplicados a regiões de clima tropical e equatorial. Neste trabalho é apresentada uma avaliação dos métodos existentes para previsão do desempenho de enlaces rádio digitais de alta capacidade, a partir da utilização de dados reais de desempenho extraídos de medidas em um tronco rádio de alta capacidade numa região tropical.

\section{Palavras-chave}

Propagação Multipercurso; Desvanecimento Seletivo; Sistema Rádio Digital; Predição de Falha. 


\section{Abstract}

Poeys Ciufo, Roque André . Effects of atmospheric multipath in line-of sight microwave systems. Rio de Janeiro, 2004. 94p. MSc. Dissertation Departamento de Engenharia Elétrica, Pontifícia Universidade Católica do Rio de Janeiro.

The variations which happen in the troposphere layers throughout the time in relation to the median condition of the signal cause various phenomena that change the received signal level at digital radio relay systems randomly. The random changes are named fading. Fading is normally classified as fast or slow. The former is normally associated with the atmospheric multipath propagation and is strongly dependent on frequency; therefore, this is named selective fading and it is normally the cause of performance degradation in high capacity digital radio relays. The existing models for statistics of multipath fading are semi empirical and based on experimental data extracts from regions the climate of which is temperate; and this gives a rough estimate with respect to the tropical and equatorial zones. This work presents an evaluation of existing methods of performance prediction for high capacity digital radio relay systems using real performance data obtained from measures of a high capacity digital radio link in operation in the tropical region.

\section{Key Words}

Multipath Propagation; Selective Fading; Digital Radio Relay Systems; Outage Prediction. 


\section{Sumário}

1 Introdução

2 Fundamentos de Propagação 15

2.1. Propagação em Espaço Livre 15

2.2. Balanço de Potência 16

2.2.1. Limiar do Receptor 16

2.2.2. Nível de Recepção Nominal 17

2.2.3. Margem de Desvanecimento 17

2.3. Refratividade e Raio Equivalente 18

$\begin{array}{ll}\text { 2.3.1. Refratividade } & 18\end{array}$

2.3.2. Raio Equivalente da Terra 20

2.4. Propagação em Visibilidade 21

2.5. Efeito das Variações da Refratividade 24

2.6. Condições Anômalas da Atmosfera 26

2.7. Desvanecimento em enlaces de micro-ondas 30

3 Metodologias para Análise de Desempenho 34

3.1. Recomendação ITU-T G.826 35

3.1.1. Camadas da Rede de Transporte 35

3.1.2. Definição e medidas dos blocos 36

3.1.3. Parâmetros e eventos de desempenho quanto a erros 36

3.1.4. Objetivos de desempenho quanto a erros (qualidade) 38

3.1.5. Divisão dos objetivos ponta a ponta da via 38

3.2. Recomendação F. 1092-1 40

3.3. Recomendação ITU-R F.695 43

3.4. Recomendação ITU-R F. 1093-1 45

3.4.1. Considerações sobre a Propagação 45

3.4.2. Contramedidas aos efeitos da propagação 46

3.4.3. Cálculo das probabilidades de falhas. 46 
3.4.3.2. Cálculo de falha usando a assinatura 48

3.5. Recomendação ITU-R P.530-10 51

3.5.1. Conceitos básicos 51

3.5.2. Perda por Propagação 52

3.5.2.1. Desvanecimento devido a multipercurso e mecanismos relacionados 53

3.5.2.1.1. Método para pequenas percentagens de tempo (desvanecimentos muito profundos) 53

3.5.2.1.2. Predição de falha não seletiva 55

3.5.3. Redução da discriminação de polarização cruzada (XPD) 55

3.5.3.1. Predição de falha por deterioração de XPD devido a efeitos que ocorrem durante condições de ar claro. $\quad 56$

3.5.4. Distorção devido aos efeitos da propagação 57

3.5.4.1. Predição de falha em sistemas digitais sem proteção 58

3.5.5. Técnicas para aliviar os efeitos da propagação por multipercurso $\quad 59$

3.5.5.1. Técnicas com diversidade $\quad 59$

3.5.5.1.1. Predição de falhas em sistemas digitais usando diversidade de espaço 60

3.5.6. Predição total de falha 62

4 Enlaces testados e metodologia 63

4.1. Levantamento e coleta dos dados de desempenho do tronco rádio 63

4.1.1. Características da localização geográfica do tronco rádio. 63

4.1.2. Topologia do tronco rádio 65

4.1.3. Características do equipamento rádio empregado 66

4.1.4. Metodologia empregada para obtenção dos dados de desempenho 67

4.2. Cálculo da predição de desempenho do tronco rádio CBA - GNA 71

5 Previsão de desempenho e análise dos resultados 75

5.1. Análise do tronco rádio baseado nas medições realizadas nas Seções de Multiplexação (SMS)

5.2. Análise do tronco rádio baseado nas medições feitas nas Seções de Regeneração (SRS) $\quad 76$

5.2.1. Cálculo do desempenho total do tronco rádio através dos resultados das Seções de Regeneração 76 
5.2.2. Análise do desempenho do tronco através das Seções de Regeneração

5.2.3. Comparação dos resultados medidos e calculados para as Seções de

Regeneração $\quad 80$

5.3. Estudo de modificações no modelo de predição de desempenho 84

5.3.1. Objetivo de SESR

5.3.2. Análise do fator geoclimático(K) 87

6 Conclusões $\quad 90$

7 Referências bibliográficas 93

8 Anexos 95

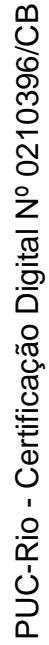




\section{Lista de figuras}

Figura 1: K mínimo versus comprimento do enlace rádio 22

Figura 2: Distribuição de probabilidades do gradiente de refratividade G 24

Figura 3: Encurvamento dos raios causado pela refração 25

Figura 4: Gradientes normais 26

Figura 5: Gradientes anômalos com gradiente de refratividade positivo (subrefração) 26

Figura 6: Gradientes anômalos com gradiente de refratividade negativo (superrefração) $\quad 27$

Figura 7: Efeito do desvanecimento plano num sinal rádio de $3590 \mathrm{MHz} \quad 31$

Figura 8: Perfil de um enlace com multipercurso 32

Figura 9: Efeito do desvanecimento rápido (seletivo) num sinal de $3590 \mathrm{MHz} 32$

Figura 10: Desvanecimento do sinal 33

Figura 11: Circuito de referência hipotético com 27500 Km 39

Figura 12:Classificação precisa do clima brasileiro $\quad 64$

$\begin{array}{ll}\text { Figura 13: Tronco CBA-GNA } & 71\end{array}$

Figura 14:Perfil do lance Serra Azul - Mineiros 72

Figura 15: Perfil do lance Serra Azul - Jataí 72

Figura 18: Gráfico linha SESR medido x SESR calculado por SRS. 82

Figura 19:Gráfico de dispersão SESR medido x SESR calculado por SRS 82

Figura 20: SESR medido x SESR calculado por SRS sem diversidade 83

Figura 21: SESR medido x SESR calculado por SRS com diversidade 83

Figura 22: Relação entre TEB e percentual de blocos errados para vários tipos de modulação (Rádio de 140 Mbps) 86

Figura 23: Curva de assinatura do rádio Alcatel 6G - $155 \mathrm{Mbps} \quad 87$

Figura 24: Comparação do fator geoclimático do modelo com o fator K corrigido

Figura 25: Comparação do fator geoclimático medido com o ajustado $\quad 89$ 


\section{Lista de tabelas}

Tabela 1: Valores importantes do fator $\mathrm{K}$ e do gradiente da refratividade $\quad 21$

Tabela 2: Bits/bloco, ESR, SESR e BBER para várias taxas de transmissão 36

Tabela 3: Objetivos de desempenho definidos na Recomendação ITU-R F.1092 42

Tabela 4: Valores de kn para vários métodos de modulação, sem equalizadores. 50

Tabela 5: Localização geográfica das estações do tronco CBA-GNA 65

Tabela 6: Comprimento dos lances do tronco CBA-GNA 66

Tabela 7: Características técnicas do rádio Alcatel 9662LH 67

Tabela 8: Análise dos dados medidos nas Seções de Multiplexação 75

Tabela 9: SESR medido em outubro 2003 por Seção de Regeneração 77

Tabela 10:Comparação SESR medido x SESR calculado pior mês. 81 